This evaluation also aimed to make further recommendations to increase use of clozapine in Mersey Care's TRS patients and assess whether there have been any differences to concerns about clozapine initiation compared to previous evaluations.

Method. An online questionnaire containing a series of Likert scales was e-mailed to all Consultant Psychiatrists in Mersey Care NHS Foundation Trust. The questionnaire asked Consultants to rate how often they felt a range of barriers interfered with successful initiation of Clozapine treatment. The barriers chosen were based on the 2019 systematic review "Barriers to using clozapine in treatment-resistant schizophrenia."

Result. Nineteen consultant psychiatrists completed the online questionnaire. All 19 indicated they either "agreed" (16\%) or "strongly agreed" (84\%) that they were confident in diagnosing TRS. This was a significant increase compared to the South London and Maudsley evaluation, with only $81 \%$ of participants in that study being "fairly familar" or "very familiar" with clozapine guidelines.

Furthermore, concerns about inadequate blood testing facilities appear to have been addressed, with no participants in this evaluation staing there were insufficient blood testing facilities. However, $53 \%$ of Consultants who completed this evaluation stated they "often" (37\%) or "very often" (16\%) have patients who refuse clozapine because of the requirement for regular blood testing. Refusal to agree to required blood testing was the commonest reason identified for failure to initiate clozapine in TRS patients. This was consistent with the results from the South London and Maudsley study.

Conclusion. Those Mersey Care consultants surveyed identified that providing patients with further information about clozapine would be the most valuable intervention to increase likelihood of uptake of clozapine in the treatment of TRS. Significant progress has been made in improving the likelihood that clozapine can be successfully initiated, especially in the removal of practitioner barriers. This evaluation suggests interventions should now be aimed at reducing patient barriers to initiation of treatment.

\section{An audit to look at the prescribing of psychotropic medication in the general adult inpatient setting in patients with emotionally unstable personality disorder}

Declan Hyland ${ }^{2}$, Charlie Daniels ${ }^{1 \star}$, Iulian Ionescu ${ }^{3}$, Christina Houghton ${ }^{4}$, Katie Goodier ${ }^{5}$ and Simon Graham ${ }^{6}$

${ }^{1}$ Core Trainee 2 in Psychiatry, Spring House, Liverpool, Mersey Care NHS Foundation Trust; ${ }^{2}$ Consultant Psychiatrist, Clock View Hospital, Liverpool, Mersey Care NHS Foundation Trust; ${ }^{3}$ Higher Trainee in Medical Psychotherapy, Spring House, Liverpool, Mersey Care NHS Foundation Trust; ${ }^{4}$ Foundation Year 2 trainee, Clock View Hospital, Liverpool, Mersey Care NHS Foundation Trust;

${ }^{5}$ Foundation Year 1 Trainee, Clock View Hospital, Liverpool, Mersey Care NHS Foundation Trust and ${ }^{6}$ Consultant in Medical Psychotherapy, Spring House, Liverpool, Mersey Care NHS

Foundation Trust

${ }^{*}$ Corresponding author.

doi: 10.1192/bjo.2021.136

Aims. To assess the frequency of prescription of psychotropic medication in patients with a primary diagnosis of emotionally unstable personality disorder (EUPD) following admission to Clock View Hospital, an inpatient unit in Mersey Care NHS Foundation Trust.

Method. A retrospective analysis of the electronic (RiO) record of 50 patients discharged from Clock View Hospital between
1 January 2020 and 1 November 2020 was performed to assess prescribing practice.

Twenty-five patients with a diagnosis of EUPD and no associated psychiatric comorbidities were included in the sample, as well as 25 patients with a diagnosis of EUPD and associated psychiatric comorbidities.

Result. $80 \%$ of the 25 patients with EUPD and associated psychiatric comorbidities were prescribed psychotropic medication prior to admission to hospital (56\% an antidepressant, $24 \%$ a mood stabiliser, $60 \%$ an antipsychotic and $8 \%$ a benzodiazepine). $64 \%$ of patients were prescribed two or more psychotropic medications. $28 \%$ were initiated on new psychotropic medications following admission. For four of the seven prescriptions commenced on psychotropic medication, prescribing practice was as advised in Mersey Care's EUPD guidelines.

Of the 25 patients with EUPD and no associated psychiatric comorbidities, $96 \%$ of the patients were prescribed psychotropic medication prior to admission to hospital (56\% an antidepressant, $20 \%$ a mood stabiliser, $72 \%$ an antipsychotic and $12 \%$ a benzodiazepine). $68 \%$ of patients were prescribed two or more psychotropic medications. Following admission, 28\% of patients were initiated on new regular psychotropic medications. For five of the eight prescriptions for new psychotropic medication, prescribing practice was as advised in Mersey Care's EUPD guidelines.

$78 \%$ of the 50 patients were prescribed as required (PRN) psychotropic medication. In 21 patients, PRN medication was prescribed for longer than one week.

Conclusion. There is a higher rate of prescribing of antipsychotic prescription in those EUPD patients with no psychiatric comorbidities compared to associated psychiatric comorbidities (72\% vs $60 \%)$. Surprisingly, there was a lower rate of psychotropic polypharmacy in those with psychiatric comorbidities.

Use of PRN psychotropic medication for longer than a week was higher in those patients with psychiatric comorbidities compared to those without psychiatric comorbidities (58\% vs 50\%). Benzodiazepines were overwhelmingly the most consistently prescribed PRN medication for patients with EUPD.

One action to consider would be highlighting the importance of trialling psychologically-minded interventions and supportive psychotherapy prior to initiation of psychotropic medication. There also needs to be consideration to use of the sedative antihistamine promethazine as a first-line PRN medication for acute agitation.

\section{Delirium diagnosis and handover to primary care providers and medical teams}

\section{Saba Inam ${ }^{1 \star}$, Joanne Flood ${ }^{2}$ and Aine Butler ${ }^{2}$}

${ }^{1}$ Highfield Healthcare, Whitehall, North Dublin Mental Health Services for Older Persons, Willow day hospital, Ashlin Centre, Beaumont Road and ${ }^{2}$ North Dublin Mental Health Services for Older Persons, Willow day hospital, Ashlin Centre, Beaumont Road ${ }^{\star}$ Corresponding author.

doi: 10.1192/bjo.2021.137

Aims. Delirium is a common medical problem with a prevalence of over $50 \%$ in over 65 s admitted to general hospitals $(1,2)$. Delirium is linked with poor clinical outcome, including increased risk of falls, prolonged admissions and an overall increased risk of morbidity and mortality $(2,3,4)$. Delirium in older adults is also associated with an increased rate of cognitive decline, future risk of cognitive decline and a risk of depression $(5,6,7)$. There is potential to improve clinical practice by improving assessment and management of delirium. It is imperative that where delirium 
is detected, it should be clearly documented to aid handover to primary care providers and medical teams $(8,9)$.

Method. The standard for this audit was set according to SIGN 157 (9). Data were collected retrospectively from consults sent to a liaison psychiatry of old age service within an acute hospital setting. The medical discharge summaries from July to December 2019 were reviewed. Two key data points were collated, the diagnoses of delirium by either medical or liaison psychiatry team and the inclusion of this diagnosis in the patient discharge summaries. An updated delirium protocol was devised and introduced in the hospital setting in January 2020 to include tools for effective diagnosis of delirium and instruction to include this diagnosis if made in patient's discharge summaries. Re-audit was initiated following the introduction of the updated delirium protocol for the period of January to March 2020.

Result. A total of 116 patients were assessed from July to December 2019. 102 discharge summaries were available for review for the purpose of this audit. Prior to the introduction of the updated delirium protocol, delirium was diagnosed by the liaison team in $57 \%$ of all referrals. Delirium was underdiagnosed by medical teams in $73 \%$ of those subsequently diagnosed. The diagnosis of delirium was present in $42 \%$ of all discharge summaries to primary care providers. Subsequent to the introduction of the updated protocol, delirium was diagnosed in $48 \%$ of all liaison referrals during the time period specified. The proportion of under-diagnosis of delirium by medical teams stayed at $73 \%$, the diagnosis of delirium was present in $53 \%$ of discharge summaries.

Conclusion. The recognition and diagnosis of delirium in the general medical setting continues to be a key issue in the management of older adults. The importance of this diagnosis and it's associated after effects needs to be disseminated amongst all care providers. Greater efforts to enhance these aspects of delirium management in the acute hospital setting are required.

\section{Lithium monitoring in patients over 65 in NHS Greater Glasgow and Clyde}

Catriona Ingram ${ }^{1 *}$, Karli Dempsey ${ }^{2}$, Gillian Scott ${ }^{3}$ and Joe Sharkey ${ }^{1}$

${ }^{1}$ NHS Greater Glasgow and Clyde; ${ }^{2}$ NHS Lanarkshire and ${ }^{3} \mathrm{NHS}$

Ayrshire and Arran

${ }^{*}$ Corresponding author.

doi: 10.1192/bjo.2021.138

Aims. Our aim was to identify current practice for Lithium monitoring for $>65 \mathrm{~s}$ in NHS GGC and assess compliance to local Lithium monitoring guidelines.

Method. A retrospective analysis was undertaken of patient data (demographics, diagnosis, biochemistry results) with Caldicott approval at two points over the course of 2018/19. For the first analysis, old age Community Mental Health Teams (CMHTs) were approached and asked to provide a list of their patients on Lithium. This was then assessed for compliance to Lithium monitoring guidelines.

For the second analysis, pharmacy provided data for every patient in the health board dispensed lithium, regardless of whether they were open to a CMHT or not. We were then able to identify patients who we had not picked up on our initial analysis, and re-assess the entire data set for compliance to Lithium monitoring guidelines.

Result. From our first analysis, 13 CMHTs identified 155 patients on Lithium. There was a high variability in how these patients were identified. $44 \%$ of patients were monitored by CMHTs who took bloods and chased them, $38 \%$ were monitored by GPs who were prompted by CMHTs in routine clinic letters, and $14 \%$ were monitored by GPs who were prompted by CMHTs more assertively using a lithium register. Overall, Lithium plasma monitoring was done well irrespective of method (91\%), however compliance to the local standards was poor (58\%) with proactive CMHT prompting GPs appearing to be the most effective method (71\%).

In our second analysis, we identified 508 patients $>65$ in NHS GGC prescribed Lithium. Of those, $44 \%$ were open to old age psychiatry, $25 \%$ general adult psychiatry and $19 \%$ were not open to anyone. Of those open to old age services, only $58 \%$ had been identified in the previous audit. Lithium monitoring compliance was better in those open to a CMHT versus those not $(61 \%$ to 23\%), and better in CMHTs where monitoring was done by CMHTs rather than GPs. For each CMHT, there were roughly 7 patients per catchment area on Lithium not open to psychiatry. Conclusion. Lithium monitoring does appear to be highly variable and not particularly compliant with local standards. CMHTs have inconsistent methods of identifying patients prescribed Lithium. There are a significant number of patients not open to old age CMHTs prescribed Lithium, and these patients have poorer compliance to Lithium monitoring. Of patients open to CMHTs, CMHT-led monitoring appears superior to other forms.

\section{A national cross-sectional survey and interviews exploring the relationship between well-being and burnout in doctors}

John Jenkins ${ }^{1 \star}$, Emma Boxley ${ }^{1}$ and Gemma Simons ${ }^{2}$

${ }^{1}$ University of Southampton and ${ }^{2}$ Centre for Workforce Wellbeing, University of southampton

${ }^{\star}$ Corresponding author.

doi: 10.1192/bjo.2021.139

Aims. Doctors' mental health is a national concern - the General Medical Council, British Medical Association and Health Education England pledge to improve their well-being. Well-being has no common definition, instead pathogenic measures such as burnout are published as a demonstration of doctors' wellbeing. Yet, the relationship between burnout and wel-being has not been explored.

Aim. to investigate the relationship between burnout and well-being.

Hypothesis. they are negatively associated, but not opposites.

Method. An online cross-sectional national survey was distributed to doctors of all grades and specialties via the Royal Colleges and doctor organisations. The Oldenburg Burnout Inventory (OLBI) measured burnout, and the Warwick-Edinburgh Mental Wellbeing Scale (WEMWBS) measured well-being. Correlation coefficients between total scores of these measures estimated the relationship. Additionally, semi-structured interviews explored personal definitions of wellbeing and its relationship with burnout. Thematic analysis was carried out.

Result. 64 doctors completed the OLBI and WEMWBS. Comparing the total scores for the questionnaires with Spearman's rho indicates a moderate negative correlation ( $r s=-0.658, p=0.00, n=64$ ). Total scores were made into binary variables, a Chi-square test showed that a low WEMWBS score $(<40)$ and a very high risk OLBI score ( $\geq 2.85$ exhaustion and $\geq 2.6$ disengagement) were statistically significantly associated $(\mathrm{X} 2(1, \mathrm{~N}=64)=4.232, \mathrm{p}=0.04)$. Three themes emerged from the 10 interviews conducted: the importance of networks/relationships outside work; scepticism towards 